\title{
Investigating the Link Between Trisomy 21 and Acquired Mutations in the GATA1 Gene
}

Triya Chakravorty, Irene Roberts

University of Oxford, MRC Weatherall Institute of Molecular Medicine, Oxford, UK Correspondence to triya.chakravorty@queens.ox.ac.uk

cite as: Chakravorty T \& Roberts I. (2020) Investigating the link between trisomy 21 and acquired mutations in the GATA1 gene. The Physician 6(1)c9 DOI: 10.38192/1.6.1.c9

\section{Background:}

Children with Down syndrome (DS) due to trisomy 21 (T21) are at an increased risk of developing the neonatal preleukaemic disorder transient abnormal myelopoiesis (TAM), which may transform into childhood acute myeloid leukaemia (ML-DS). Leukaemic cells in TAM and ML-DS have acquired mutations in the GATA1 gene. Although it is clear that acquired mutations in GATA1 are necessary for the development of TAM and ML-DS, questions remain concerning the mechanisms of disease. TAM mostly resolves spontaneously within three months; only $\sim 1$ in 10 cases progress to ML-DS, and this transformation always occurs before age four years. This suggests that the foetal cellular environment created by T21 may be important for the acquisition of GATA1 mutations.

Methods:

To investigate the importance of the foetal cell context in TAM and ML-DS, GATA1 mutational analysis was carried out using next generation deep sequencing on blood samples collected from DS children enrolled into the Oxford-Imperial Down Syndrome Cohort Study.

Results:

Here it is shown that children with GATA1 mutations present at birth do not acquire additional new mutations in GATA1 in the first four years, and children without GATA1 mutations at birth do not subsequently acquire mutations in GATA1.

Conclusions:

The results suggest that the foetal cellular environment in T21 is critical for the acquisition of GATA1 mutations. It is likely that GATA1 mutant clones require factor(s) present specifically in the foetal liver microenvironment to sustain their proliferation and survival. Furthermore, screening for GATA1 mutations at birth in DS children is likely to be a reliable way of identifying those at reduced risk of developing ML-DS. 